\title{
Massive Sequencing: A New Tool for the Control of Alcoholic Fermentation in Wine?
}

\author{
Dimitrios Kioroglou (D), Jessica LLeixá, Albert Mas (i) and Maria del Carmen Portillo * \\ Facultat d'Enologia, Department Bioquímica i Biotecnologia, Universitat Rovira i Virgili, 43003 Tarragona, Spain; \\ dimitrios.kioroglou@urv.cat (D.K.); jessica.lleixa@urv.cat (J.L.); albert.mas@urv.cat (A.M.) \\ * Correspondence: carmen.portillo@urv.cat; Tel.: +34-977-558688
}

Received: 23 December 2017; Accepted: 24 January 2018; Published: 26 January 2018

\begin{abstract}
In wine industry, there is a prevalent use of starter cultures to promote a controlled and efficient alcoholic fermentation preventing the growth of spoilage microbes. However, current trends in enology aim to combine the guaranteed success of monitored process and the complexity of fermentations either by inoculating autochthonous starters or by performing spontaneously to produce distinctive wines. To understand the complex roles of microorganisms on wine fermentation, we must understand their population dynamics and their relationships with wine quality and metabolome. Current metagenomics techniques based on massive sequencing are gaining relevance to study the diversity and evolution of microbial population on every stage of the wine making process. This new tool and technique increases the throughput and sensitivity to study microbial communities. This review focuses on the current knowledge about wine alcoholic fermentation, the contribution of massive sequencing techniques and the possibility of using this tool for microbial control.
\end{abstract}

Keywords: next-generation-sequencing; alcoholic fermentation; bioinformatics; high-throughput sequencing; wine; microbiota; metagenomics

\section{Introduction}

Wine is an alcoholic beverage with great cultural and economic importance, which results from the alcoholic fermentation process. During this process, yeasts derive energy by consuming sugars that occur naturally in the grapes and at the same time produce ethanol and carbon dioxide as byproducts [1]. From the yeasts genera the most widely used, due to its fermentation capacities, is the yeast Saccharomyces [1], whereas non-Saccharomyces yeasts contribute to wine flavor, although they can also spoil wines [2-4].

In addition to the different fermentation techniques used currently by the industry, the characteristics of the wine also depend upon other factors such as climate, soil and grape variety where variation of these factors attribute to the distinctiveness of the wine [5]. Moreover, the interplay between the wine microbiota and the microbiota of the fermentation facilities has been verified but not completely understood [6]. Due to this complexity of interactions between microorganisms in the wine itself, during fermentation, but also between wine microbiota and environment, the wine industry has adopted the use of starter cultures as a mean of control and quality improvement [7]. Nevertheless, more in-depth knowledge in needed in order to understand how microbial interactions may affect the wine quality.

The diversity of the vineyard and grape microbiota has been long ago investigated via traditional microbiological techniques involving agar plate cultivation, microscopy and biochemical characterization, focusing primarily on identifying pathogenic microorganisms or microorganisms that have been associated with wine spoilage. Nevertheless, these techniques fail to identify unculturable microorganisms that comprise a considerable fraction of the wine microbiota $[8,9]$. 
Nowadays, molecular techniques such as qPCR (quantitative polymerase chain reaction) and PCR-DGEE (polymerase chain reaction denaturing gradient gel electrophoresis) are widely used for detection and monitoring of microbial communities in wine. The former technique is more appropriate for detection and monitoring of a desired microorganism, whereas the latter for microbial community profiling. Although both techniques are supplemented with culture-dependent methods, however PCR-DGEE fails to detect species in low abudance, and qPCR suffers from scalability problems when many strains should be targeted $[10,11]$.

These drawbacks of the aforementioned culture-dependent and molecular techniques come to solve recent novel techniques that are based on massive sequencing, and which in recent years have been regarded as the tool of choice for studying microbial communities during the various stages of alcoholic fermentation. Although there have been encouraging findings demonstrating the superiority of the massive sequencing over the classical methods concerning speed, sensitivity and accuracy, however most of the research has been confined to describing the constituents microorganisms and their abundance fluctuation over time. Therefore, the aim of this review, apart from exhibiting the contribution of massive sequencing to monitoring alcoholic fermentation, is to demonstrate the possibility of using this method as a tool for microbial control.

\section{Sequencing Methods}

The metagenomic analysis of wine samples is mainly performed via amplicon-based sequencing which through marker-genes amplification facilitates the taxonomic and phylogenetic profiling of the microbiome [12]. After the Sanger sequencing, that was developed back in 1977 by Sanger et al. [13] and was considered the first generation sequencing, second (SGS) and third (TGS) generation sequencing, collectivelly referred as next-generation sequencing (NGS), have been introduced in research as fast and cost-effective solutions.

Despite the fact that TGS solves many of the disadvantages of the SGS, still is under development and not widely applied in research. From the area of SGS, which is based on "sequencing by synthesis" method, the most popular platforms will be presented.

\subsection{Ion Torrent}

Introduced back in 2010 [14], Ion Torrent sequences the template DNA strand by detecting hydrogen ions that are released during the polymerization process. As a technology, with an error rate of $1.71 \%$ [15], it does not require modified nucleotides and it generates reads of around $200 \mathrm{bp}$ in length allowing for multiple runs and more data generation [16].

\subsection{Pyrosequencing}

The most recent variant of pyrosequencing, 454 pyrosequencing, was introduced back in 2005 [17], and was the first affordable platform allowing whole genome sequencing. As a technology, it relies on the light signal detection that is emitted after the release of phosphate during the incorporation of a nucleotide by the DNA polymerase. With an error rate below 1\% [18] 454 pyrosequencing is capable of generating reads of over $400 \mathrm{bp}$ in length [19].

\subsection{Illumina}

With the first Illumina sequencer being available back in 2006, Illumina technology is based on the usage of fluorescently labeled dNTP terminators and the detection of light signal upon incorporation. Recent Illumina machines, HiSeq and MiSeq, have decreased the error rate below $1 \%$ and are capable of generating reads of around $300 \mathrm{bp}$ in length [20].

From the aforementioned platforms, Illumina is the most widely used, with $52 \%$ of the published research citing it, followed by pyrosequencing that holds $48 \%$ of the total citations [21]. However, pyrosequencing technology has been discontinued, and currently Illumina is being considered as the largest contributor to SGS. 


\section{Amplified Genomic Regions}

Apart from choosing the most appropriate sequencing platform, researchers have to decide the genomic region that is going to be used for the taxonomic classification of the metagenomic wine sample. As far as bacteria are concerned, the 16S ribosomal RNA (rRNA) gene is the common target that is used in research for taxonomic assignment.

The 16S rRNA gene contains nine hypervariable regions (V1-V9), which all have been used as potential classification targets generating different results. For instance, Bokulich et al. [22] used the $\mathrm{V} 4$ and V5 domain so as to ascertain which one is the most taxonomically informative for profiling bacterial communities. Based on the results, the V4 domain was regarded as more suitable for profiling lactic acid bacteria (LAB), as it gave more taxonomic depth comparing to the V5 domain.

Campisano et al. [23] used a 700 bp region that includes the domains from V5 up to V9 in order to assess the impact of pest management on bacterial endophytic communities of Merlot and Chardonnay grapevines, with the results indicating abundance differences of operational taxonomic units (OTUs) between organic and intergrated pest management (IPM) grapevines. The same genomic region was also targeted by Perazzolli et al. [24] in a study of leaf microbiota, that resulted in identification of beneficial microbial communities that could be used as a tool for crop protection. In the same manner, in the past years other researchers have been focusing on other domains for classification purposes. For instance, Sundquist et al. [25] favored the domains V1,V2 and V4, Liu et al. [26] the domains V2, V3 and V4, and Chakravorty et al. [27] the domains V2 and V3.

Regarding fungal classification, researchers have also displayed variability concerning genomic region preference. For instance, David et al. [28] sequenced the 18S rRNA gene to show that 454 pyrosequencing is much more reliable than classical techniques for studying yeast communities in alcoholic fermentation. Holland et al. [29] pyrosequenced the D1-D2 regions of the 26S rRNA, demonstrating that changes in arbuscular mycorrhizal fungal communities do not depend on irrigation frequency. Bokulich and Mills [30] targeted the IT1, ITS2 and the whole ITS in order to compare their classification efficiency by utilizing a mock community. Although they favored the IT1 region, nevertheless they urged for caution as none of these regions reconstructed reliably the whole mock community. Encouraging results targeting the ITS region have been yielded also from the researches of Pinto et al. [31] and Stefanini et al. [32] indicating this region as a suitable target for yeast classification.

\section{Bioinformatic Tools}

Regardless the NGS platform a researcher decides to utilize, the sequencing of wine metagenomic samples generates a significant amount of data that necessitate the use of bioinformatic pipelines. Despite the plethora of bioinformatic tools available, the most widely used will be presented.

\subsection{QIIME}

QIIME, which stands for Quantitative Insights Into Microbial Ecology, is a bioinformatic package, offering a variety of microbial community analyses and visualizations, that wraps other software packages with python code [33]. Some of the most frequent wrapped applications include mothur [34], blast [35], PyNAST (Python Nearest Alignment Space Termination) [36], RDP (Ribosomal Database Project) Classifier [37], FastTree [38] and USEARCH (unique word count search) [39].

\subsection{MOTHUR}

Mothur is a bioinformatic package that re-implements in $\mathrm{C}$ and $\mathrm{C}^{++}$code other software packages removing that way any external dependecies during installation. Some of the re-implemented algorithms include DOTUR (Distance-Based OTU and Richness), SONS (Shared OTUs and Similarity), TreeClimber, LIBSHUFF, and UniFrac, and additioanlly the mothur team has incorporated its own analytical features to the platform [34]. 


\subsection{MG-RAST}

MG-RAST, which stands for Metagenomics Rapid Annotation using Subsystem Technology, is a server based platform with initial aim the annotation of complete or draft microbial genomes [40]. Currently, MG-RAST offers an automated solution for phylogenetic classification and functional classification of metagenomic samples.

A comparison of these three bioinformatic pipelines has been conducted by Plummer et al. [41] using 16S rRNA gut microbial data. The study concluded that all of the three pipelines were able to generate similar and reliable results with common limitation the ability to classify at the species level due to the type of data. The main differences between the pipelines concerned the usability and duration of analysis. MG-RAST is a more user friendly pipeline compared to the command-line based QIIME and MOTHUR, whereas QIIME required approximately $1 \mathrm{~h}$ to complete the analysis with MOTHUR and MG-RAST $10 \mathrm{~h}$ and 2 days respectively.

\section{Databases}

One of the most crucial steps of metagenomic analysis is the taxonomic classification of the microbial community. Apart from other factors, such as the sequence length, the parameters used for quality filtering and the implemented algorithm, this step can be greatly influenced by the chosen database. Currently, there are a number of highly curated databases available, such as Greengenes for 16S rRNA [42], SILVA for small (16S/18S, SSU) and large (23S/28S, LSU) subunit rRNA [43], UNITE for ITS region [44] and RDP for 16S and 28S rRNA classification [45]. However, classification based on these databases should be regarded as a rough estimation of the microbial composition as genera abundances or even taxonomic assignments can be greatly influenced by the chosen percentage of homology.

\section{Analysis of Alcoholic Fermentation}

There are numerous studies dedicated to the microbial analysis of wine alcoholic fermentation, but until now great focus has been given on describing microbial abundance succession during the various stages of alcoholic fermentation. These studies have attested the superiority of NGS over classical methods [28] and offered novel insights into the microbial communities.

Although bacteria are not directly connected to wine quality, acetic acid bacteria (AAB) and lactic acid bacteria (LAB) play a significant role to the final wine product. Portillo and Mas [46], in a Grenache variety wine fermentation study, showed that $A A B$ and $L A B$ are more abundant than previously thought, with a dominance of Gluconobacter during the mid fermentation. The latter finding contradicts the previous notion that Gluconobacter, being alcohol sensitive, usually declines during the alcoholic fermentation [47-49]. Similar results have also been yielded in other studies of low-sulfited or unsulfited wine fermentations [50].

Additionally, NGS analysis has created the notion that apart from AAB, other bacteria, not previously described, may be present during the process. Support to this hypothesis came from Godálová et al. [51] in a study of Blaufränkisch and Grüner Veltliner vines, where in addition to genera already found in other studies, such as Sphingomonas, Variovorax, Pantoea, Enterobacter and Tatumella, new genera were detected, namely Amycolatopsis, Hydrogenophilus, Snodgrassella, Telluria, Gilliamella, Lelliottia, and Lonsdale quercina. However, the possible impact of these newly described genera is still to be demonstrated.

Other studies come to supplement existing ones. For instance, Bokulich et al. [22] showed that Acetobacter, Gluconobacter, and Gluconoacetobacter are dominant in winemaking processes, whereas Campanaro et al. [52] in a grape marc study added that Gluconobacter and Gluconoacetobacter do not survive a prolonged grape marc storage period.

Moreover, the empirically based concept of the coined term "terroir", that is distinction of wine quality due to regional features, has been put under the prism of NGS analysis and verified recently. 
Results from Zarraonaindia et al. [53] suggested that the soil serves as a bacterial reservoir for the vines and subsequently Bokulich et al. [54], in a 200 commercial wine fermentations study, demonstrated the correlation of wine microbiota, wine performance and wine metabolome. These authors even predicted the metabolome of the wine from the microbial composition by using machine learnig techniques [54]. Similar results have also been generated from other studies $[55,56]$.

Besides bacteria, NGS analysis has also given significant insights into the yeast population during fermentation. The most frequent fungi described by NGS analysis are Saccharomyces, Hanseniaspora, Issatchenkia, Rhodotorula, Penicillium, Cladosporium, Botrytis, Sporobolomyces, Aspergillus, Cryptococcus and Pichia [56-58], with most studies reporting high abundance of Hanseniaspora and Saccharomyces during the mid and end of the fermentation respectively. Stefanini et al. [32] in a Vino Santo study, found that fungal species composition undergoes a dynamic change with a declining tendency overtime, and that small changes in fermentation procedures may result in significant differences in microbial communities. As advocates to these findings come older studies that have demonstrated that aerobic yeasts are the first to decrease in abundance, and that the mid fermentation yeast genera, such as Hanseniaspora, Candida, Metschnikowia and Torulaspora, cannot be not recovered on plates at high ethanol concentration in presence of Saccharomyces [59,60]. Interestingly, S. cerevisiae, found in very low abundance at the beginning of the fermentation, manages to rise in dominance at the end of the it. In accordance to this, Lleixà et al. [61] drew a comparison between the dynamics of Saccharomyces cerevisiae and Hanseniaspora vineae after inoculation in Macabeo and Merlot grape varieties. The results indicated that fermentation of $S$. cerevisiae inoculated must was faster than the one with $H$. vineae inoculation, and that inoculation with $S$. cerevisiae is necessary as $H$. vineae alone leads to incomplete alcoholic fermentation. However $H$. vineae was able to dominate the microbiota in Macabeo must but not the Merlot perhaps due to high exhibited yeast diversity of Merlot must.

Another important question that NGS analysis has been called to answer, is whether grapes are the source of spoilage microorganisms [62], or the wine-making equipment [63]. Even though there is no clear answer to this debate, studies from Suárez et al. [64] and Pinto et al. [56] seem to support the latter hypothesis.

\section{Control of Alcoholic Fermentation}

Controlling the alcoholic fermentation of wine-making is a very complex process. Unlike fed-batch alcoholic fermentation in bioreactors, where algorithms have been developed for the estimation of parameters that may lead to higher biomass concentrations and yield of a specific compound [65], wine alcoholic fermentation incorporates higher order of complexity, as it concerns (i) the determination of all the microbial composition throughout the fermentation process; (ii) the comprehension of the interplay between different microbial communities; (iii) the definition of a series of metabolites that contribute to the wine quality, and (iv) the integration of all these information into a predictive machine-learning model.

In the past, a series of studies have set the ground for controlling alcoholic fermentation by monitoring or modifying certain fermentation parameters, but most of the results were empirical and their interpretation was not an easy task. Various studies have shown that yeasts increase their production of volatile compounds at low fermentation temperatures [66-68]. Therefore, wine-makers that aim at enhancing wine aroma could take advantage of this factor. Another popular method, is choosing a specific yeast strain for improving specific aspects of the wine, with studies having used this technique so as to improve wine characteristics of Sauvignon [69] and Chardonnay [70]. Furthermore, addition of certain nutrients that will prevent the fermentation from stucking, is a common practice. For instance, Cramer et al. [71] developed a fermentation kinetic model which showed that fermentation rate can be increased upon addition of ammonium salts, whereas Birch et al. [72] supported that yeast growth rate and sugar degradation could be influenced by magnesium concentrations. On the other hand, adaptive evolution approaches are aiming towards the creation of non-recombinant yeast strains that could modify wine characteristics, as for instance in 
the study of McBryde et al. [73]. Additionally non-S. cerevisiae yeasts are known of adding distinct flavors to the wine but due to the fact that they can easily become replaced by $S$. cerevisiae, authors such as Soden et al. [74] have suggested the use of mixed cultures controlled by sequential inoculation.

Although all the above practices are means of manipulating specific aspects of wine fermentation towards a specific outcome, they treat alcoholic fermentation as a black-box without controlling the microbial composition of the wine and consequently the wine quality consistency they are aiming to provide may not be certain. NGS analysis is aiming to tackle these obstacles, but as a relatively new approach so far has yielded descriptive results on the bacteria and yeast genera abundances that have been encountered during the various fermentation stages. Until now, studies from the food industry have already evinced this type of analysis as a promising strategy for the detection of previously undescribed spoiler bacteria $[75,76]$, underlying its suitability for controlling alcoholic fermentation. Nevertherless, NGS analysis has as an intrinsic difficulty the overwhelming amount of metagenomic analysis tools, machine-learning algorithms, databases and parameters that the researcher has to choose from. Because small changes of parameters may result in singificantly altered taxonomic assignment results [26], a possible solution may come from the use of mock communities datasets with known species compositions [77]. This strategy has already been implemented in studies such as the one by Bokulich et al. [78] in order to compare the performance of different classifiers. Even though mock communities datasets cannot lead to the development of a standardized NGS analysis with fixed parameters, as metagenomic samples are bound to laboratory protocols, NGS platforms, environmental and grape variety differences, they may nonetheless serve as a way to validate the robustness of a bioinformatic pipeline or as a starting point for the subsequent metagenomic analysis.

Setting a solid ground for metagenomic analysis is of paramount importance, so additional analyses such as metatranscriptomics and metabolomics can function as determinant factors for the development of system-biology networks aiming for the understanding of microbial communities interaction, and machine-learning prediction models focusing on the quality of the final wine product. With encouraging results coming from studies such as the one by Bokulich et al. [54] where it has been demonstrated that microbial composition of grape must can predict wine metabolome, the future of controlling alcoholic fermentation via NGS analysis seems nothing but promising.

\section{Conclusions}

The aim of this review is to cite contemporary contributions of massive sequencing techniques to wine alcoholic fermentation, and the possibility of being used as a tool for microbial control. Wine alcoholic fermentation is a complex process that encompasses an intricate and dynamic interaction between microbial populations that leads towards the composition of a wine metabolome that defines the final wine quality and characteristics.

As a way of controlling alcoholic fermentation, the industry has adopted various techniques, such as starter cultures and process monitoring and modification, but these approaches rely on empirical results as little is known about the relationships within the wine microbiome and its correlation to the final wine product.

High-throughput sequencing, based on NGS platforms, has been presented as a metagenomic analysis tool that offers higher speed, accuracy and taxonomic resolution compared to classical culture-dependent and molecular techniques. Till now, the implementation of this technology has yielded significant yet descriptive research results on microbial dynamics in connection to the fermentation stages. Although, NGS metagenomic analysis comprises a vast amount of bioinformatic tools, databases and machine-learning algorithms, however publicly available mock communities datasets may serve as ways of algorithm benchmarking, robustness check of bioinformatic pipelines, and parameters initialization.

These mock communities and highly curated taxonomic databases could set a solid foundation for the metagenomic analysis, upon which metatranscriptomics and metabolomics will be based and provide all the necessary knowledge for the development of system-biology networks and 
prediction models for deciphering microbial population dynamics and prediction of final wine product, correspondingly. Regarding the latter, research has provided encouraging results highlighting the potential and benefits of massive sequencing as a tool for controlling alcoholic fermentation.

Acknowledgments: This study received financial support by the co-funding of regional, national and international programme (COFUND) Martí Franquès Fellowship (2017MFP-COFUND-7), and the Spanish Government AGL2015-73273-JIN (AEI/FEDER/EU).

Author Contributions: Dimitrios Kioroglou, Albert Mas and Maria del Carmen Portillo conceived the review. Dimitrios Kioroglou, Jessica LLeixá, Albert Mas and Maria del Carmen Portillo wrote the paper.

Conflicts of Interest: The authors declare no conflict of interest.

\section{References}

1. Ribéreau-Gayon, P.; Dubourdieu, D.; Donèche, B.; Lonvaud, A. Handbook of Enology, the Microbiology of Wine and Vinifications; John Wiley \& Sons: Hoboken, NJ, USA, 2006; Volume 1.

2. Carrau, F.M.; Medina, K.; Boido, E.; Farina, L.; Gaggero, C.; Dellacassa, E.; Versini, G.; Henschke, P.A. De novo synthesis of monoterpenes by Saccharomyces cerevisiae wine yeasts. FEMS Microbiol. Lett. 2005, $243,107-115$.

3. Varela, C.; Siebert, T.; Cozzolino, D.; Rose, L.; McLean, H.; Henschke, P. Discovering a chemical basis for differentiating wines made by fermentation with 'wild' indigenous and inoculated yeasts: Role of yeast volatile compounds. Aust. J. Grape Wine Res. 2009, 15, 238-248.

4. Ciani, M.; Comitini, F.; Mannazzu, I.; Domizio, P. Controlled mixed culture fermentation: A new perspective on the use of non-Saccharomyces yeasts in winemaking. FEMS Yeast Res. 2010, 10, 123-133.

5. Belda, I.; Zarraonaindia, I.; Perisin, M.; Palacios, A.; Acedo, A. From Vineyard Soil to Wine Fermentation: Microbiome Approximations to Explain the "terroir" Concept. Front. Microbiol. 2017, 8, doi:10.3389/fmicb.2017.00821.

6. Bokulich, N.A.; Ohta, M.; Richardson, P.M.; Mills, D.A. Monitoring Seasonal Changes in Winery-Resident Microbiota. PLoS ONE 2013, 8, e66437.

7. Spano, G.; Torriani, S. Editorial: Microbiota of Grapes: Positive and Negative Role on Wine Quality. Front. Microbiol. 2016, 7, 2036, doi:10.3389/fmicb.2016.02036.

8. Amann, R.I.; Ludwig, W.; Schleifer, K.H. Phylogenetic identification and in situ detection of individual microbial cells without cultivation. Microbiol. Rev. 1995, 59, 143-169.

9. Curtis, T.P.; Sloan, W.T.; Scannell, J.W. Estimating prokaryotic diversity and its limits. Proc. Natl. Acad. Sci. USA 2002, 99, 10494-10499.

10. Andorrà, I.; Landi, S.; Mas, A.; Esteve-Zarzoso, B.; Guillamón, J.M. Effect of fermentation temperature on microbial population evolution using culture-independent and dependent techniques. Food Res. Int. 2010, 43, 773-779.

11. Prakitchaiwattana, C.J.; Fleet, G.H.; Heard, G.M. Application and evaluation of denaturing gradient gel electrophoresis to analyse the yeast ecology of wine grapes. FEMS Yeast Res. 2004, 4, 865-877.

12. Franzosa, E.A.; Hsu, T.; Sirota-Madi, A.; Shafquat, A.; Abu-Ali, G.; Morgan, X.C.; Huttenhower, C. Sequencing and beyond: Integrating molecular 'omics' for microbial community profiling. Nat. Rev. Microbiol. 2015, 13, 360-372.

13. Sanger, F.; Nicklen, S.; Coulson, A.R. DNA sequencing with chain-terminating inhibitors. Proc. Natl. Acad. Sci. USA 1977, 74, 5463-5467.

14. Rusk, N. Torrents of sequence. Nat. Methods 2011, 8, 44, doi:10.1038/nmeth.f.330.

15. Quail, M.A.; Smith, M.; Coupland, P.; Otto, T.D.; Harris, S.R.; Connor, T.R.; Bertoni, A.; Swerdlow, H.P.; Gu, Y. A tale of three next generation sequencing platforms: Comparison of Ion Torrent, Pacific Biosciences and Illumina MiSeq sequencers. BMC Genom. 2012, 13, 341, doi:10.1186/1471-2164-13-341.

16. Gupta, A.K.; Gupta, U. Next generation sequencing and its applications. In Animal Biotechnology; Elsevier: Amsterdam, The Netherlands, 2014; pp. 345-367.

17. Margulies, M.; Egholm, M.; Altman, W.E.; Attiya, S.; Bader, J.S.; Bemben, L.A.; Berka, J.; Braverman, M.S.; Chen, Y.J.; Chen, Z.; et al. Genome sequencing in microfabricated high-density picolitre reactors. Nature 2005, 437, 376-380. 
18. Morozova, O.; Marra, M.A. Applications of next-generation sequencing technologies in functional genomics. Genomics 2008, 92, 255-264.

19. Pettersson, E.; Lundeberg, J.; Ahmadian, A. Generations of sequencing technologies. Genomics 2009, 93, 105-111.

20. Bleidorn, C. Third generation sequencing: Technology and its potential impact on evolutionary biodiversity research. Syst. Biodivers. 2016, 14, 1-8.

21. Morgan, H.H.; Du Toit, M.; Setati, M.E. The grapevine and wine microbiome: Insights from high-throughput amplicon sequencing. Front. Microbiol. 2017, 8, doi:10.3389/fmicb.2017.00820.

22. Bokulich, N.A.; Joseph, C.L.; Allen, G.; Benson, A.K.; Mills, D.A. Next-generation sequencing reveals significant bacterial diversity of botrytized wine. PLOS ONE 2012, 7, e36357.

23. Campisano, A.; Antonielli, L.; Pancher, M.; Yousaf, S.; Pindo, M.; Pertot, I. Bacterial endophytic communities in the grapevine depend on pest management. PLOS ONE 2014, 9, e112763.

24. Perazzolli, M.; Antonielli, L.; Storari, M.; Puopolo, G.; Pancher, M.; Giovannini, O.; Pindo, M.; Pertot, I. Resilience of the natural phyllosphere microbiota of the grapevine to chemical and biological pesticides. Appl. Environ. Microbiol. 2014, 80, 3585-3596.

25. Sundquist, A.; Bigdeli, S.; Jalili, R.; Druzin, M.L.; Waller, S.; Pullen, K.M.; El-Sayed, Y.Y.; Taslimi, M.M.; Batzoglou, S.; Ronaghi, M. Bacterial flora-typing with targeted, chip-based Pyrosequencing. BMC Microbiol. 2007, 7, 108, doi:10.1186/1471-2180-7-108.

26. Liu, Z.; DeSantis, T.Z.; Andersen, G.L.; Knight, R. Accurate taxonomy assignments from $16 \mathrm{~S}$ rRNA sequences produced by highly parallel pyrosequencers. Nucleic Acids Res. 2008, 36, e120, doi:10.1093/nar/gkn491.

27. Chakravorty, S.; Helb, D.; Burday, M.; Connell, N.; Alland, D. A detailed analysis of 16S ribosomal RNA gene segments for the diagnosis of pathogenic bacteria. J. Microbiol. Methods 2007, 69, 330-339.

28. David, V.; Terrat, S.; Herzine, K.; Claisse, O.; Rousseaux, S.; Tourdot-Maréchal, R.; Masneuf-Pomarede, I.; Ranjard, L.; Alexandre, H. High-throughput sequencing of amplicons for monitoring yeast biodiversity in must and during alcoholic fermentation. J. Ind. Microbiol. Biotechnol. 2014, 41, 811-821.

29. Holland, T.C.; Bowen, P.; Bogdanoff, C.; Hart, M. Arbuscular mycorrhizal fungal communities associated with Vitis vinifera vines under different frequencies of irrigation. Am. J. Enol. Vitic. 2014, 65, 222-229.

30. Bokulich, N.A.; Mills, D.A. Improved selection of internal transcribed spacer-specific primers enables quantitative, ultra-high-throughput profiling of fungal communities. Appl. Environ. Microbiol. 2013, 79, 2519-2526.

31. Pinto, C.; Pinho, D.; Sousa, S.; Pinheiro, M.; Egas, C.; Gomes, A.C. Unravelling the diversity of grapevine microbiome. PLOS ONE 2014, 9, e85622.

32. Stefanini, I.; Albanese, D.; Cavazza, A.; Franciosi, E.; De Filippo, C.; Donati, C.; Cavalieri, D. Dynamic changes in microbiota and mycobiota during spontaneous 'Vino Santo Trentino' fermentation. Microb. Biotechnol. 2016, 9, 195-208.

33. Caporaso, J.G.; Kuczynski, J.; Stombaugh, J.; Bittinger, K.; Bushman, F.D.; Costello, E.K.; Fierer, N.; Peña, A.G.; Goodrich, J.K.; Gordon, J.I.; et al. QIIME allows analysis of high-throughput community sequencing data. Nat. Methods 2010, 7, 335-336.

34. Schloss, P.D.; Westcott, S.L.; Ryabin, T.; Hall, J.R.; Hartmann, M.; Hollister, E.B.; Lesniewski, R.A.; Oakley, B.B.; Parks, D.H.; Robinson, C.J.; et al. Introducing mothur: Open-source, platform-independent, community-supported software for describing and comparing microbial communities. Appl. Environ. Microbiol. 2009, 75, 7537-7541.

35. Altschul, S.F.; Gish, W.; Miller, W.; Myers, E.W.; Lipman, D.J. Basic local alignment search tool. J. Mol. Biol. 1990, 215, 403-410.

36. Caporaso, J.G.; Bittinger, K.; Bushman, F.D.; DeSantis, T.Z.; Andersen, G.L.; Knight, R. PyNAST: A flexible tool for aligning sequences to a template alignment. Bioinformatics 2009, 26, 266-267.

37. Wang, Q.; Garrity, G.M.; Tiedje, J.M.; Cole, J.R. Naive Bayesian classifier for rapid assignment of rRNA sequences into the new bacterial taxonomy. Appl. Environ. Microbiol. 2007, 73, 5261-5267.

38. Price, M.N.; Dehal, P.S.; Arkin, A.P. FastTree 2-approximately maximum-likelihood trees for large alignments. PLOS ONE 2010, 5, e9490. 
39. Edgar, R.C. Search and clustering orders of magnitude faster than BLAST. Bioinformatics 2010, 26, $2460-2461$.

40. Meyer, F.; Paarmann, D.; D'Souza, M.; Olson, R.; Glass, E.M.; Kubal, M.; Paczian, T.; Rodriguez, A.; Stevens, R.; Wilke, A.; et al. The metagenomics RAST server-A public resource for the automatic phylogenetic and functional analysis of metagenomes. BMC Bioinform. 2008, 9, 386, doi:10.1186/1471-2105-9-386.

41. Plummer, E.; Twin, J.; Bulach, D.M.; Garland, S.M.; Tabrizi, S.N. A comparison of three bioinformatics pipelines for the analysis of preterm gut microbiota using $16 \mathrm{~S}$ rRNA gene sequencing data. J. Proteom. Bioinform. 2015, 8, 283, doi:10.4172/jpb.1000381.

42. DeSantis, T.Z.; Hugenholtz, P.; Larsen, N.; Rojas, M.; Brodie, E.L.; Keller, K.; Huber, T.; Dalevi, D.; Hu, P.; Andersen, G.L. Greengenes, a chimera-checked $16 \mathrm{~S}$ rRNA gene database and workbench compatible with ARB. Appl. Environ. Microbiol. 2006, 72, 5069-5072.

43. Quast, C.; Pruesse, E.; Yilmaz, P.; Gerken, J.; Schweer, T.; Yarza, P.; Peplies, J.; Glöckner, F.O. The SILVA ribosomal RNA gene database project: Improved data processing and web-based tools. Nucleic Acids Res. 2012, 41, D590-D596.

44. Abarenkov, K.; Henrik Nilsson, R.; Larsson, K.H.; Alexander, I.J.; Eberhardt, U.; Erland, S.; Høiland, K.; Kjøller, R.; Larsson, E.; Pennanen, T.; et al. The UNITE database for molecular identification of fungi-recent updates and future perspectives. New Phytol. 2010, 186, 281-285.

45. Cole, J.R.; Wang, Q.; Fish, J.A.; Chai, B.; McGarrell, D.M.; Sun, Y.; Brown, C.T.; Porras-Alfaro, A.; Kuske, C.R.; Tiedje, J.M. Ribosomal Database Project: Data and tools for high throughput rRNA analysis. Nucleic Acids Res. 2013, 42, D633-D642.

46. Portillo, M.; Mas, A. Analysis of microbial diversity and dynamics during wine fermentation of Grenache grape variety by high-throughput barcoding sequencing. LWT Food Sci. Technol. 2016, 72, 317-321.

47. Du Toit, W.; Lambrechts, M. The enumeration and identification of acetic acid bacteria from South African red wine fermentations. Int. J. Food Microbiol. 2002, 74, 57-64.

48. González, Á.; Hierro, N.; Poblet, M.; Mas, A.; Guillamón, J.M. Application of molecular methods to demonstrate species and strain evolution of acetic acid bacteria population during wine production. Int. J. Food Microbiol. 2005, 102, 295-304.

49. Joyeux, A.; Lafon-Lafourcade, S.; Ribéreau-Gayon, P. Evolution of acetic acid bacteria during fermentation and storage of wine. Appl. Environ. Microbiol. 1984, 48, 153-156.

50. Bokulich, N.A.; Swadener, M.; Sakamoto, K.; Mills, D.A.; Bisson, L.F. Sulfur dioxide treatment alters wine microbial diversity and fermentation progression in a dose-dependent fashion. Am. J. Enol. Vitic. 2015, $66,73-79$.

51. Godálová, Z.; Kraková, L.; Puškárová, A.; Bučková, M.; Kuchta, T.; Piknová, L.; Pangallo, D. Bacterial consortia at different wine fermentation phases of two typical Central European grape varieties: Blaufränkisch (Frankovka modrá) and Grüner Veltliner (Veltlínske zelené). Int. J. Food Microbiol. 2016, 217, 110-116.

52. Campanaro, S.; Treu, L.; Vendramin, V.; Bovo, B.; Giacomini, A.; Corich, V. Metagenomic analysis of the microbial community in fermented grape marc reveals that Lactobacillus fabifermentans is one of the dominant species: Insights into its genome structure. Appl. Microbiol. Biotechnol. 2014, 98, 6015-6037.

53. Zarraonaindia, I.; Owens, S.M.; Weisenhorn, P.; West, K.; Hampton-Marcell, J.; Lax, S.; Bokulich, N.A.; Mills, D.A.; Martin, G.; Taghavi, S.; et al. The soil microbiome influences grapevine-associated microbiota. mBio 2015, 6, doi:10.1128/mBio.02527-14.

54. Bokulich, N.A.; Collins, T.S.; Masarweh, C.; Allen, G.; Heymann, H.; Ebeler, S.E.; Mills, D.A. Associations among wine grape microbiome, metabolome, and fermentation behavior suggest microbial contribution to regional wine characteristics. mBio 2016, 7, e00631-16, doi:10.1128/mBio.00631-16.

55. Knight, S.; Klaere, S.; Fedrizzi, B.; Goddard, M.R. Regional microbial signatures positively correlate with differential wine phenotypes: Evidence for a microbial aspect to terroir. Sci. Rep. 2015, 5, doi:10.1038/srep14233.

56. Pinto, C.; Pinho, D.; Cardoso, R.; Custódio, V.; Fernandes, J.; Sousa, S.; Pinheiro, M.; Egas, C.; Gomes, A.C. Wine fermentation microbiome: A landscape from different Portuguese wine appellations. Front. Microbiol. 2015, 6, doi:10.3389/fmicb.2015.00905. 
57. Bokulich, N.A.; Thorngate, J.H.; Richardson, P.M.; Mills, D.A. Microbial biogeography of wine grapes is conditioned by cultivar, vintage, and climate. Proc. Natl. Acad. Sci. USA 2014, 111, E139-E148.

58. Setati, M.E.; Jacobson, D.; Bauer, F.F. Sequence-based analysis of the Vitis vinifera L. cv Cabernet Sauvignon grape must mycobiome in three South African vineyards employing distinct agronomic systems. Front. Microbiol. 2015, 6, doi:10.3389/fmicb.2015.01358.

59. Beltran, G.; Torija, M.J.; Novo, M.; Ferrer, N.; Poblet, M.; Guillamón, J.M.; Rozès, N.; Mas, A. Analysis of yeast populations during alcoholic fermentation: A six year follow-up study. Syst. Appl. Microbiol. 2002, 25, 287-293.

60. Combina, M.; Elía, A.; Mercado, L.; Catania, C.; Ganga, A.; Martinez, C. Dynamics of indigenous yeast populations during spontaneous fermentation of wines from Mendoza, Argentina. Int. J. Food Microbiol. 2005, 99, 237-243.

61. Lleixà, J.; Martín, V.; Portillo, M.d.C.; Carrau, F.; Beltran, G.; Mas, A. Comparison of fermentation and wines produced by inoculation of Hanseniaspora vineae and Saccharomyces cerevisiae. Front. Microbiol. 2016, 7, doi:10.3389/fmicb.2016.00338.

62. Renouf, V.; Claisse, O.; Lonvaud-Funel, A. Understanding the microbial ecosystem on the grape berry surface through numeration and identification of yeast and bacteria. Aust. J. Grape Wine Res. 2005, 11, 316-327.

63. Couto, J.A.; Neves, F.; Campos, F.; Hogg, T. Thermal inactivation of the wine spoilage yeasts Dekkera/Brettanomyces. Int. J. Food Microbiol. 2005, 104, 337-344.

64. Suárez, R.; Suárez-Lepe, J.; Morata, A.; Calderón, F. The production of ethylphenols in wine by yeasts of the genera Brettanomyces and Dekkera: A review. Food Chem. 2007, 102, 10-21.

65. Selişteanu, D.; Petre, E.; Roman, M.; Ionete, C.; Popescu, D. Estimation strategies for kinetic parameters of an alcoholic fermentation bioprocess. In Proceedings of the IEEE SICE Annual Conference, Taipei, Taiwan, 18-21 August 2010; pp. 3560-3565.

66. Killian, E.; Ough, C. Fermentation esters-Formation and retention as affected by fermentation temperature. Am. J. Enol. Vitic. 1979, 30, 301-305.

67. Mc Lellan, M.; Mclellan, M.R. The effect of fermentation temperature on chemical and sensory characteristics of wines from seven white grape cultivars grown in New York State. Am. J. Enol. Vitic. 1986, 37, 190-194.

68. Torija, M.J.; Beltran, G.; Novo, M.; Poblet, M.; Guillamón, J.M.; Mas, A.; Rozes, N. Effects of fermentation temperature and Saccharomyces species on the cell fatty acid composition and presence of volatile compounds in wine. Int. J. Food Microbiol. 2003, 85, 127-136.

69. Dubourdieu, D.; Tominaga, T.; Masneuf, I.; des Gachons, C.P.; Murat, M.L. The role of yeasts in grape flavor development during fermentation: The example of Sauvignon blanc. Am. J. Enol. Vitic. 2006, 57, 81-88.

70. Eglinton, J.M.; Mcwilliam, S.J.; Fogarty, M.W.; Francis, I.L.; Kwiatkowski, M.J.; Høj, P.B.; Henschke, P.A. The effect of Saccharomyces bayanus-mediated fermentation on the chemical composition and aroma profile of Chardonnay wine. Aust. J. Grape Wine Res. 2000, 6, 190-196.

71. Cramer, A.C.; Vlassides, S.; Block, D.E. Kinetic model for nitrogen-limited wine fermentations. Biotechnol. Bioeng. 2002, 77, 49-60.

72. Birch, R.M.; Ciani, M.; Walker, G.M. Magnesium, calcium and fermentative metabolism in wine yeasts. J. Wine Res. 2003, 14, 3-15.

73. McBryde, C.; Gardner, J.M.; de Barros Lopes, M.; Jiranek, V. Generation of novel wine yeast strains by adaptive evolution. Am. J. Enol. Vitic. 2006, 57, 423-430.

74. Soden, A.; Francis, I.; Oakey, H.; Henschke, P. Effects of co-fermentation with Candida stellata and Saccharomyces cerevisiae on the aroma and composition of Chardonnay wine. Aust. J. Grape Wine Res. 2000, 6, 21-30.

75. De Filippis, F.; La Storia, A.; Stellato, G.; Gatti, M.; Ercolini, D. A selected core microbiome drives the early stages of three popular Italian cheese manufactures. PLoS ONE 2014, 9, e89680.

76. Chaillou, S.; Chaulot-Talmon, A.; Caekebeke, H.; Cardinal, M.; Christieans, S.; Denis, C.; Desmonts, M.H.; Dousset, X.; Feurer, C.; Hamon, E.; et al. Origin and ecological selection of core and food-specific bacterial communities associated with meat and seafood spoilage. ISME J. 2015, 9, 1105-1118. 
77. Bokulich, N.A.; Rideout, J.R.; Mercurio, W.G.; Shiffer, A.; Wolfe, B.; Maurice, C.F.; Dutton, R.J.; Turnbaugh, P.J.; Knight, R.; Caporaso, J.G. Mockrobiota: A public resource for microbiome bioinformatics benchmarking. mSystems 2016, 1, e00062-16, doi:10.1128/mSystems.00062-16.

78. Bokulich, N.A.; Rideout, J.R.; Kopylova, E.; Bolyen, E.; Patnode, J.; Ellett, Z.; McDonald, D.; Wolfe, B.; Maurice, C.F.; Dutton, R.J.; et al. A Standardized, Extensible Framework for Optimizing Classification Improves Marker-Gene Taxonomic Assignments; Technical Report; PeerJ PrePrints; 2015. Available online: https: / / doi.org/10.7287/peerj.preprints.934v2 (accessed on 25 January 2018).

(C) 2018 by the authors. Licensee MDPI, Basel, Switzerland. This article is an open access article distributed under the terms and conditions of the Creative Commons Attribution (CC BY) license (http:/ / creativecommons.org/licenses/by/4.0/). 